\title{
Determination of SMN1 and SMN2 Copy Numbers in a Korean Population using Multiplex Ligation-dependent Probe Amplification
}

\author{
Seoyoung Yoon ${ }^{1, *}$, Chang Hoon Lee ${ }^{2, *}$, and Kyung-A Lee ${ }^{1}$ \\ Department of Laboratory Medicine', Yonsei University College of Medicine, Seoul; Department of Laboratory Medicine², \\ Konkuk University School of Medicine, Seoul, Korea
}

\begin{abstract}
Determination of the copy number of the survival motor neuron (SMN) gene is important for detecting spinal muscular atrophy (SMA) carriers and compound heterozygous patients. Multiplex ligationdependent probe amplification (MLPA) assay is a simple and efficient technique used for detecting variations in the copy numbers of different genes. Race- and ethnicity-based variation in the SMA carrier frequency and the '2+0' genotype of SMN1 are important factors that should be considered when estimating the risk of being an SMA carrier. Since SMN2 plays a disease-modifying role, accurate determination of SMN2 copy numbers in SMA patients can serve as a useful prognostic tool. Therefore, information on the SMN2 genotype distributions in normal populations will be helpful in selecting appropriate reference samples for MLPA analysis. To determine SMA carrier frequencies and SMN genotype distribution, we determined the copy numbers of SMN1 and SMN2 genes using the MLPA assay in 100 unrelated Korean individuals with no family history of SMA. The frequency of SMA carriers in the Korean population appears to be 1 in 50 , which indicates that the prevalence of SMA among Koreans is the same as that among individuals in the Western countries. Two of the 100 normal individuals enrolled in this study showed 3 copies of the SMN1 gene. Therefore, 1.0\% of the 198 normal alleles in this population was estimated to be 2-copy alleles ('2+0' genotype). SMN2 copy numbers showed a high degree of individual variation. Our results showed that $64 \%$ of the individuals had 2 copies of SMN2, but 36\% individuals had between 0, 1, or 3 copies of the gene. (Korean J Lab Med 2010;30:93-6)
\end{abstract}

Key Words : Spinal muscular atrophy, Multiplex ligation-dependent probe amplification, Carrier, Copy number, Survival motor neuron gene, Koreans

Spinal muscular atrophy (SMA), a disease characterized by the degeneration of the anterior horn cells of the spinal cord, causes symmetric proximal muscle weakness. In approximately 95\% of cases, SMA is caused by the homozygous deletion of the survival motor neuron 1 (SMN1)

Received : September 4, 2009

Manuscript No : KJLM09-112

Revision received: November 20, 2009

Accepted: November 20, 2009

Corresponding author : Kyung-A Lee, M.D.

Department of Laboratory Medicine, Yonsei University College of Medicine, Gangnam Severance Hospital, 712 Eonju-ro, Gangnam-gu, Seoul 135-720, Korea

Tel : +82-2-2019-3531 Fax : +82-2-3462-9483

E-mail : KAL1119@yuhs.ac

*The first 2 authors contributed equally to this paper.

*This study was supported by Konkuk University. gene (5q13) or its conversion to SMN2 [1]. The prevalence of SMA, an autosomal recessive disease, is approximately 1 in 10,000 newborns [2]. Hendrickson et al. [3] reported that the carrier frequency of SMN mutation was different among different ethnic groups in North America: the carrier frequency was $2.7 \%$ in Caucasians, $1.8 \%$ in Asians, $1.1 \%$ in African Americans, and $0.8 \%$ in Hispanics. The carrier frequency of the later 2 groups was lower than that of the former groups. Copy number analysis of the SMN1 gene is important to identify carriers with SMN mutation. The American College of Medical Genetics (ACMG) recommends universal screening for the presence of SMA mutation to identify carriers because this condition is as- 
sociated with clinically severe presentation and a high carrier frequency [4]. Since SMA occurs in all populations, regardless of race or ethnicity, carrier testing should be recommended to all couples considering pregnancy or in early stages of pregnancy. However, carrier testing for SMA, using SMN1 gene analysis, has not been considered as a part of routine clinical investigation in such couples in Korea.

SMN1 gene produces full-length transcripts only whereas the majority of SMN2 transcripts lack exon 7 due to alternative splicing. In normal subjects, most of the functional SMN protein is a result of the SMN1 gene, with only a very small amount coming from the SMN2 gene. In more than $95 \%$ of patients, SMA is due to the deletion of SMN1 or its conversion into $S M N 2$, while in about $5 \%$ of the patients, the disease is caused by intragenic point mutations of SMN1. SMN2 has been reported to play a diseasemodifying role because the SMN2 copy numbers inversely correlate with the severity of the SMA phenotype. Various methods have been reported for determining the copy numbers of the SMN gene; these methods include denatured high-performance liquid chromatography (DHPLC) and real-time quantitative polymerase chain reaction (PCR) [5-8]. Real-time PCR is difficult to set up because the multiplexing required to evaluate a useful number of loci can be difficult to optimize. DHPLC has been successfully used in screening for SMA carriers; however, when performed by inexperienced technicians, this technique yields inconsistent results. The multiplex ligation-dependent probe amplification (MLPA) assay is efficient in the detection of copy number changes in various genes [3]. Reports indicate that the MLPA assay is better and more efficient than the 2-step DHPLC. The MLPA assay provides the unique ability to hybridize several probes specific for SMN1 and SMN2 genes in a single experiment [9].

Although SMA is considered to be a panethnic disease, the epidemiologic data for a specific ethnic group should be collected since the frequency of the carriers can significantly differ among various ethnic groups [3, 10]. We assessed the copy numbers of SMN1 and SMN2 genes among Koreans using the MLPA assay to estimate the SMA car- rier frequencies and to determine SMN genotype distributions that are required for genetic risk assessment.

We collected blood samples from 100 unrelated Korean individuals who had no family history of SMA after written informed consent was obtained from each subject. MLPA analysis was performed using kits P021 according to the recommendations of the manufacturer (MRC Holland, Amsterdam, Netherlands). The PCR products were analyzed by using a 310 ABI sequencer (Applied Biosystems, Foster City, CA, USA). Analysis was performed using the GeneMarker software, version 1.6 (Softgenetics, State College, PA, USA).

The results were classified according to the copy numbers of SMN1 and SMN2 (Table 1). The frequency of SMA carriers $(2.0 \%)$ determined in this study is similar to that observed in previous studies conducted in Western countries and Korea [3, 5, 10]. The copy number of SMN1 can vary on a chromosome. Two of the 100 individuals investigated in this study showed 3 copies of the SMN1 ' $2+1$ ' genotype (Fig. 1). Therefore, 198 were normal alleles (including 2 normal alleles in the individuals with 1 copy of SMN1, 192 normal alleles in the individuals with 2 copies of SMN1, and 4 normal alleles in individuals with 3 copies of SMN1 (the assuming $2+1$ genotype)); this shows that $1 \%$ of the alleles of these 198 normal alleles were of the '2+0' genotype.

Thus, a carrier may possess 2 copies of the gene on 1 chromosome and have 0 copies on another chromosome,

Table 1. SMN1 and SMN2 copy numbers in a Korean population

\begin{tabular}{lcc}
\hline SMN1:SMN2 ratio & No. of subjects & Interpretation \\
\hline $1: 1$ & 1 & SMA carrier \\
$1: 3$ & 1 & SMA carrier \\
$2: 0$ & 2 & Noncarrier \\
$2: 1$ & 28 & Noncarrier \\
$2: 2$ & 63 & Noncarrier \\
$2: 3$ & 3 & Noncarrier \\
$3: 2$ & 1 & Noncarrier \\
$2: 2 / 3: 1^{\star}$ & 1 & \\
Total & 100 & \\
\hline
\end{tabular}

*Hybrid SMN genes, SMN1 exon 7:SMN2 exon 7/SMN1 exon 8:SMN2 exon 8.

Abbreviations: SMN, survival motor neuron; SMA, spinal muscular atrophy. 

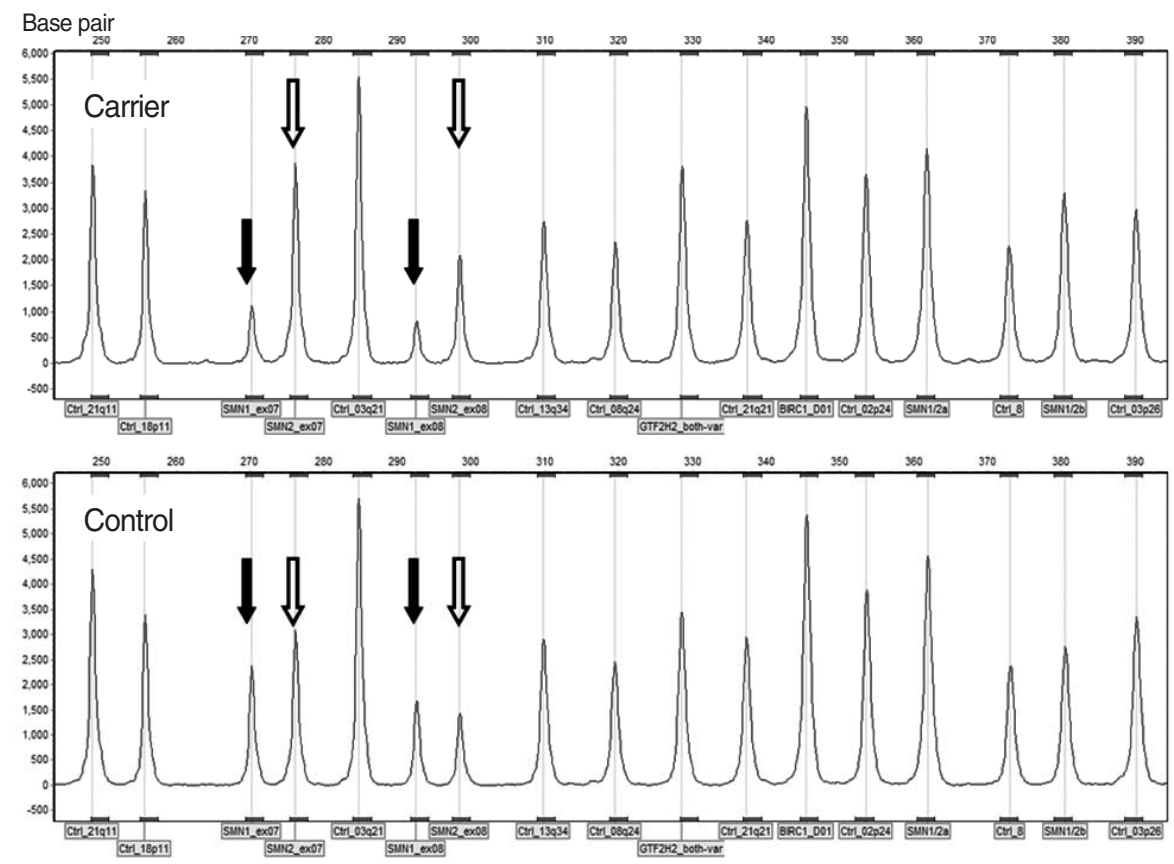

Fig. 1. Electrophoreogram of SMN1: $S M N 2=1: 3$. The carrier is heterozygous for the deletion of the SMN1 gene as shown by reduction in peak heights from 2 probes for exons 7 and 8 (closed arrows). The relative peak height ratios of SMN2 gene increase to approximately 1.5 , indicating the presence of 3 copies of SMN2 (open arrows).

which can lead to a false-negative result when testing for SMA carrier [11]. In this light, the $2+0$ genotype is an essential factor that needs to be considered when calculating the risk of being an SMA carrier, the carrier frequency, and the rates of de novo mutation. Therefore, the estimation of the frequency of the 3-copy SMN1 genotype in the Korean population is essential for a more reliable assessment of genetic risk. Lee et al. [5] have reported that in a Korean population, the frequency for 3-copy SMN1 genotype, calculated using real-time PCR, was approximately $19 \%$. Huang et al. [12] conducted a study on a Taiwanese population using MLPA and reported the frequency of 3copy SMN1 to be 7.1\%. Our study, which used the same assay, yielded similar results in Korean population $(P$ value=0.08).

We also assessed the SMN2 copy numbers in this study population, and observed a high degree of individual variation. Sixty-four percent of the individuals had 2 copies of SMN2, but $36 \%$ had 0, 1, or 3 copies of this gene. Since a higher copy number of the SMN2 has an inverse relation with the severity of SMA [2], the accurate identification of SMN2 copy numbers can serve as a useful tool in predicting the prognosis of patients with SMA. Although the SMN2 gene number assay would be helpful in a clini- cal setting, the performance standard for this assay has not yet been established. The most important shortcoming of the SMN2 assay is the lack of adequate controls for its validation. To determine whether there are any variations in SMN2 copy numbers, MLPA peak profiles of patients should be compared with those of reference samples with normal copy numbers. For example, if we compare the MLPA peak pattern of an SMA patient with 2 copies of SMN2 with that of the reference sample with 1 copy of SMN2, the SMN2 copy number in the patient will show a false-positive increase. Thus, our results of the SMN2 genotype distributions will facilitate accurate selection of reference samples with 2 copies of SMN2 in the Korean population.

In summary, race- and ethnicity-based variation in the carrier frequency and the ' $2+0$ ' genotype are important factors that should be considered when estimating the risk of being an SMA carrier. In addition, de novo mutation rates and intragenic SMN mutations have to be evaluated. The SMA carrier frequency in the Korean population appears to be 1 in 50, which indicates the prevalence of SMA in Korea is similar to that in the Western countries. The frequency of individuals with the ' $2+0$ ' genotype was estimated to be $1.0 \%$. In MLPA analysis for determina- 
tion of variations in SMN2 copy numbers, it is important to use appropriate reference samples with normal copy numbers.

\section{REFERENCES}

1. Lefebvre S, Burglen L, Reboullet S, Clermont O, Burlet P, Viollet L, et al. Identification and characterization of a spinal muscular atrophy-determining gene. Cell 1995;80:155-65.

2. Ogino $S$ and Wilson RB. Spinal muscular atrophy: molecular genetics and diagnostics. Expert Rev Mol Diagn 2004;4:15-29.

3. Hendrickson BC, Donohoe C, Akmaev VR, Sugarman EA, Labrousse $\mathrm{P}$, Boguslavskiy L, et al. Differences in SMN1 allele frequencies among ethnic groups within North America. J Med Genet 2009; 46:641-4.

4. Prior TW. Carrier screening for spinal muscular atrophy. Genet Med 2008;10:840-2.

5. Lee TM, Kim SW, Lee KS, Jin HS, Koo SK, Jo I, et al. Quantitative analysis of SMN1 gene and estimation of SMN1 deletion carrier frequency in Korean population based on real-time PCR. J Korean Med Sci 2004;19:870-3.

6. Su YN, Hung CC, Li H, Lee CN, Cheng WF, Tsao PN, et al. Quantitative analysis of SMN1 and SMN2 genes based on DHPLC: a highly efficient and reliable carrier-screening test. Hum Mutat 2005;25:
460-7.

7. van der Steege G, Grootscholten PM, van der Vlies P, Draaijers TG, Osinga J, Cobben JM, et al. PCR-based DNA test to confirm clinical diagnosis of autosomal recessive spinal muscular atrophy. Lancet 1995;345:985-6.

8. Wang CC, Chang JG, Jong YJ, Wu SM. Universal multiplex PCR and $C E$ for quantification of SMN1/SMN2 genes in spinal muscular atrophy. Electrophoresis 2009;30:1102-10.

9. Scarciolla O, Stuppia L, De Angelis MV, Murru S, Palka C, Giuliani $\mathrm{R}$, et al. Spinal muscular atrophy genotyping by gene dosage using multiple ligation-dependent probe amplification. Neurogenetics 2006;7:269-76.

10. Cusin V, Clermont O, Gerard B, Chantereau D, Elion J. Prevalence of SMN1 deletion and duplication in carrier and normal populations: implication for genetic counselling. J Med Genet 2003;40:e39.

11. Ogino S, Wilson RB, Gold B. New insights on the evolution of the SMN1 and SMN2 region: simulation and meta-analysis for allele and haplotype frequency calculations. Eur J Hum Genet 2004;12: 1015-23.

12. Huang $\mathrm{CH}$, Chang YY, Chen $\mathrm{CH}$, Kuo YS, Hwu WL, Gerdes T, et al. Copy number analysis of survival motor neuron genes by multiplex ligation-dependent probe amplification. Genet Med 2007;9: 241-8. 